Phase-3-Studie mit PARP1-Inhibitor BSI-201 zur Behandlung von metastasiertem dreifach negativem Brustkrebs angelaufen

Sanofi-Aventis und dessen hundertprozentige Tochter BiPar Sciences haben im Dezember 2009 bekannt gegeben, dass die klinische Prüfung des PARP1-Inhibitors BSI-201 zur Behandlung von metastasiertem triple-negativem Brustkrebs (mTNBC) planmäßig voranschreitet. Die Phase-3-Studie erfüllt die Erwartungen hinsichtlich der Patienten- und Zentrenrekrutierung in den USA. Die Prüfärzte der Studie bestätigen die erfolgreiche Rekrutierung eines Großteils der Patienten.

BSI-201 ist im Juli 2009 in den USA in eine klinische Phase-3-Studie eingetreten und wird bei Patientinnen mit mTNBC in Kombination mit Chemotherapie untersucht. BSI-201 ist eine neue, noch in der klinischen Erprobung befindliche zielgerichtete Therapie, die die Poly(ADPRibose)Polymerase (PARP1) hemmt, ein Enzym, das an der DNA-Reparatur beteiligt ist. Die Entscheidung, im Juli 2009 mit der Phase-3Studie zu beginnen, basierte auf den ermutigenden Phase-2-Studienergebnissen, die im Mai 2009 auf dem internationalen Krebskongress ASCO vorgestellt worden waren. In der klinischen Phase-2-Studie erhielten Frauen mit mTNBC randomisiert Gemcitabin und Carboplatin (GC) in Kombination mit der Prüfsubstanz BSI-201 oder nur GC. Aktualisierte Phase2-Daten - einschließlich des Gesamtüberlebens - wurden im Dezember 2009 bei einer Postersitzung anlässlich des 32. Annual San Antonio Breast Cancer Symposiums vorgestellt. Danach erhöhte die zusätzliche Verabreichung von BSI201 in Kombination mit GC das mittlere Gesamtüberleben von 7,7 Monaten auf 12,2 Mo- nate $(\mathrm{HR}=0,5, \mathrm{p}=0,005)$. Im BSI-201-Arm waren weder Häufigkeit noch Schweregrad chemotherapieassoziierter unerwünschter Ereignisse erhöht.

Die US-amerikanische Food and Drug Administration (FDA) hat der Substanz BSI-201 zur Behandlung des mTNBC ein beschleunigtes Prüfverfahren bewilligt. Diese Regelung der FDA erlaubt die beschleunigte Bereitstellung von Substanzen gegen schwerwiegende Erkrankungen.

Weitere Informationen bei Sanofi-Aventis Deutschland GmbH Judith Kramer

Brand \& Scientific Communications Industriepark Höchst Gebäude K 703 65926 Frankfurt am Main

Tel. +49 69 305-84412, Fax -18802

presse@sanofi-aventis.com

\section{Biosimilars in der Supportivtherapie}

\section{HEXAL baut Onkologie-Portfolio aus}

Mit Epoetin alfa HEXAL ${ }^{\circledR}$ bietet das Holzkirchener Unternehmen neben Filgrastim HEXAL $^{\circledR}$ nun bereits das zweite Biosimilar für die onkologische Supportivtherapie. Bei dosisintensiven oder dosisdichten Chemotherapien kommt es häufig zu Komplikationen, etwa einer febrilen Neutropenie und/oder einer Chemotherapie-assoziierten Anämie. «Ohne Granulozytenkoloniestimulierende Faktoren (G-CSF) könnten wir viele Patienten gar nicht oder nicht zeitgerecht behandeln», sagt Dr. Peter Borchmann, Universitätsklinikum Köln. Und Professor Hans Tesch, Frankfurt/M., ergänzt: «Die EpoetinGabe ist unverzichtbar - vorausgesetzt die Indikation wird streng gestellt und der $\mathrm{Hb}$ Ziel-Wert von maximal $12 \mathrm{~g} / \mathrm{dl}$ beachtet.»
Eine Lanze für Sicherheit und Vergleichbarkeit der Biosimilars mit den Referenzprodukten bricht Professor Fritz Sörgel, Leiter des Instituts für Biomedizinische und Pharmazeutische Forschung (IBMP). «Bedenken sind ungerechtfertigt. Die Kritik, die Biosimilars seit ihrer Markteinführung begleitet, ist nicht nur haltlos, sondern kommt einer Infragestellung der Europäischen Arzneimittelagentur (EMEA) gleich.» Dass die Biosimilars von HEXAL den Referenzprodukten weder qualitativ noch in Sicherheitsaspekten nachstehen, legt Dr. Carsten Brockmeyer, Leiter Entwicklung Biotechnologie der HEXAL AG, sehr eindrücklich dar. Die Gleichwertigkeit mit den Referenzprodukten wurde durch umfangreiche analytische und klinische Studien belegt. Für alle Herstellungsschritte werden neue technische Verfahren einge- setzt. Aus der Supportivtherapie sind Biosimilars nicht mehr wegzudenken, so das Fazit der Expertenrunde auf einer Pressekonferenz von HEXAL, die im Rahmen des 29. Deutschen Krebskongresses in Berlin stattgefunden hat. Die Behandlungsergebnisse seien überzeugend, und der Preis sei fair.

Quelle

Pressekonferenz «Biosimilars in der Supportivtherapie», 25.02.2010, veranstaltet von HEXAL im Rahmen des 29. Deutschen Krebskongresses in Berlin.

Weitere Informationen bei

Ulrike Hartmann

Medical Manager Biosimilars

über Hexal Kundenservice

Tel.: +498004392527

\title{
PharmaTicker+++ PharmaTicker+++ PharmaTicker+++ PharmaTicker+++
}

MSD Sharp \& Dohme GmbH. Ein antiemetisches Dreierregime mit Aprepitant bietet Patienten mit hämatologischen Neoplasien einen effektiveren Schutz vor Übelkeit und Erbrechen als die konventionelle Emesis-Prophylaxe mit Ondansetron und Dexamethason. Dies zeigten die Ergebnisse einer Phase-III-Studie, die die gute antiemetische Wirksamkeit und Verträglichkeit von Aprepitant bei myeloablativen Hochdosisregimen zur Vorbereitung auf eine HSCT belegten.

MSD Sharp \& Dohme GmbH

Anette Menzel

Tel. +49 89 4561-1353, Fax -1329

anette_menzel@msd.de
Qiagen GmbH. Pfizer und DxS (eine hundertprozentige Tochter der QIAGEN N.V.), gaben im Februar eine Vereinbarung zur Entwicklung eines begleitenden Diagnostik-Tests für PF-04948568 (CDX-110) bekannt - ein in der Entwicklung befindlicher Impfstoff zur Immuntherapie für die Behandlung von Glioblastoma multiforme (GBM). Der Wirkstoff befindet sich aktuell in Phase II der klinischen Entwicklung für die Behandlung von neu diagnostiziertem GBM.

Public Relations Qiagen GmbH

Dr. Thomas Theuringer

Tel. +4921032911826
Novartis Pharma GmbH. Die EMA (European Medicines Agency) hat das von Novartis eingereichte Zulassungsdossier für die Therapie mit Nilotinib $\left(\right.$ Tasigna $^{\circledR}{ }^{\circledR}$ ) bei Patienten mit neu diagnostizierter chronischer myeloischer Leukämie (CML) in der chronischen Phase angenommen. Die EMA prüft nun die Zulassung dieser Therapieoption für die Erstlinientherapie der CML.

Novartis Pharma GmbH

Dr. Irene Roth

Tel. +49 911273 12643, Fax -11020

irene.roth@ novartis.com 